\section{Retinal detachment repair and cataract surgery in patients with atopic dermatitis}

\begin{abstract}
Purpose The aim of this study is to report a case series of ocular complications including retinal detachment (RD) and cataract in atopic dermatitis (AD) and surgical management involving a majority of Caucasian patients. Patients and methods This study is an observational case series, originally designed as an audit. It involves detailed discussion of history, clinical features, and surgical management of patients presenting with retinal detachment and cataracts secondary to severe AD. Six consecutive patients with diagnosis of severe $\mathrm{AD}$ requiring posterior segment and cataract surgery were included in the study. Results Eight eyes of six patients had retinal detachment. Most of them involved the temporal retina. The retinal breaks were located anteriorly close to the ora serrata in six eyes and Giant tear retinal (GRT) detachment was found in two eyes. Five eyes had proliferative vitreo-retinopathy (PVR) at presentation. All six patients had bilateral cataracts and cataract surgery was performed in eleven eyes. Bilateral simultaneous surgery was essential in two patients. Three eyes had secondary intra-ocular lens (IOL) implantation with pars plana vitrectomy for subluxed lens implant.

Conclusions Anterior retinal breaks and temporal RD are common in retinal detachment secondary to AD. PVR is often present, which makes surgical management difficult. Cataract formation is quite often and late subluxation of IOL may occur.

Eye (2017) 31, 1296-1301; doi:10.1038/eye.2017.151; published online 11 August 2017
\end{abstract}

Department of Ophthalmolgy, Royal Victoria Hospital, Belfast, UK

Correspondence:

$\checkmark$ Gnana Jothi, Department of Ophthalmolgy, Royal

Victoria Hospital, Belfast BT12 6BA, UK

Tel: +44 2890632342;

Fax: +44 2890634684

E-mail: gjvasuki@yahoo.com

Received: 31 December 2016

Accepted in revised form:

22 June 2017

Published online:

11 August 2017

\section{Introduction}

Retinal detachment associated with atopic dermatitis was first reported in 1937 by Balyeat. ${ }^{1}$ Most of the literature available regarding this condition is from an Oriental study population.
V Gnana Jothi, S McGimpsey, JA Sharkey and WC Chan
Only case reports in Caucasian population are available in literature ${ }^{2}$ and they date to the pre-vitrectomy era. Retinal surgery and management of complex cataract has evolved hugely, with improvement in technology and equipment. In the recent years, the incidence of Eczema has increased by $41.8 \%$ and prevalence has increased by $48.2 \%$ in England. ${ }^{3}$ Our study is an observational case series which aims to report the nature of retinal detachment (RD), cataract and related problems in atopic dermatitis (AD) patients in local population. Cataract in atopic dermatitis is a well known entity. We also aim to highlight the complexities in managing these patients who need surgical intervention to manage cataract, retinal detachment and dislocation of intra-ocular lens (IOL).

\section{Materials and methods}

This study is a retrospective, consecutive observational case series and was conducted in a tertiary care centre (Royal Victoria Hospital, Belfast) during the period of 2015-2016 as an audit. Case notes and electronic care records were reviewed to gather clinical details, as some patients attended more than one hospital. We discuss in detail about the presentation, clinical features, and surgical management of these patients presenting with RD, cataracts and related clinical problems secondary to severe AD. Six consecutive adult patients with diagnosis of severe $\mathrm{AD}$ requiring posterior segment and cataract surgery were included in the study. Posterior segment surgery included retinal detachment repair and corrective surgery for dislocated IOL.

\section{Case 1}

Nineteen-year-old oriental male, presented with seeing a shadow in his right eye for two weeks. He had asthma and AD. Visual acuity was 6/6 
in his right eye and 6/5 in his left eye. Bilateral minimal posterior sub-capsular cataracts were present. Fundus examination showed bilateral temporal RD with multiple holes anteriorly close to the ora serrata and chorio-retinal atrophic changes. Right eye had PVR with sub-retinal fibrotic bands (Figure 1) and an asymptomatic shallow RD was present in his left eye.

The RD in his right eye was repaired with a scleral buckle procedure, and a decision was made to observe the left eye. Two years later there was worsening of the cataract in his right eye, and progression of the RD in his left eye. This correlated with a period of flaring of his symptoms due to AD. Scleral buckle procedure was performed in his left eye for the RD and cataract surgery in his left eye subsequently after few months. This patient also had history of tapping and rubbing both his eyes for symptom relief due to AD from childhood days. His vision remains $6 / 6$ in his right eye and $6 / 5$ in his left eye with a stable retina after nine years of follow-up.

\section{Case 2}

A twenty-one-year-old Caucasian male, presented to the eye department with bilateral blurring of vision for six weeks. Severe atopic dermatitis needing systemic immune supression was a major concern. Azathioprine was initiated after failed Cyclosporine by the Dermatologist. Oral and topical steroids were used on multiple occasions for brief periods to control the symptoms. Unusual iris changes were present in his right eye, which did not fit with any particular diagnosis (Figure 2). He had bilateral white cortical and posterior sub-capsular cataracts. Ultrasound did not show any retinal detachment in either eye. Cataract surgery was performed in his Left eye and a multifocal IOL was placed in the bag. Retinal examination on day one after surgery revealed a giant tear retinal (GRT) related detachment with four clock hours of retinal detachment superotemporally. GRT was repaired as a two stage procedure. Stage one involved pars plana vitrectomy (PPV), perfluorooctane tamponade, endolaser and cryopexy. One week later bilateral simultaneous surgery —cataract surgery in the right eye with a multifocal IOL and stage two of RD repair in his left eye was undertaken. Stage two was removal of the heavy liquids and gas tamponade with $20 \%$ sulphur hexa-fluoride. Vision was restored to $6 / 9$ unaided in both eyes post surgery

(Figure 3). On further questioning retrospectively, patient admitted having flashing lights in his left eye just prior to cataract surgery. Constant itching and eye rubbing were noticed during every consultation. He described vigorous eye rubbing to relieve the itching. The iris changes was most likely due to self inflicted trauma to the eyes as there
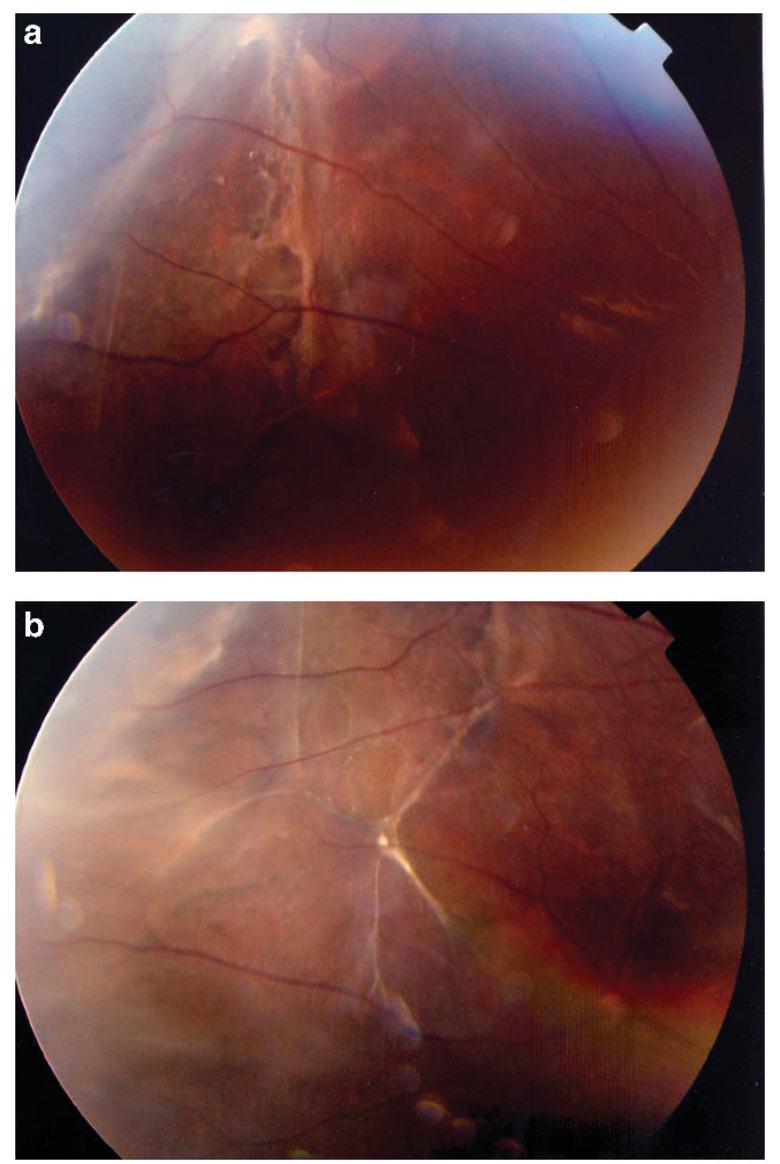

Figure 1 Sub-retinal fibrosis secondary to PVR at initial presentation.

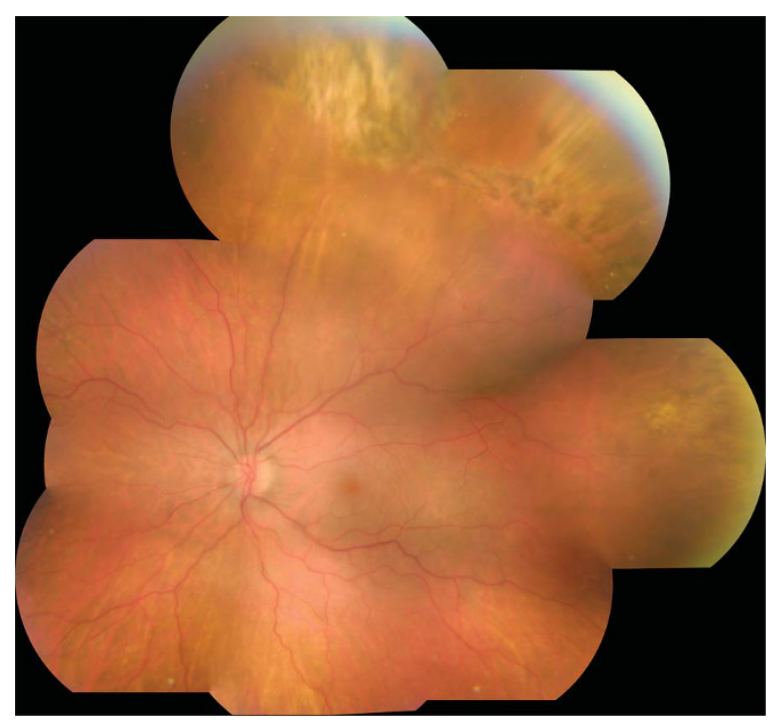

Figure 2 Post GRT repair in a patient with severe AD. 


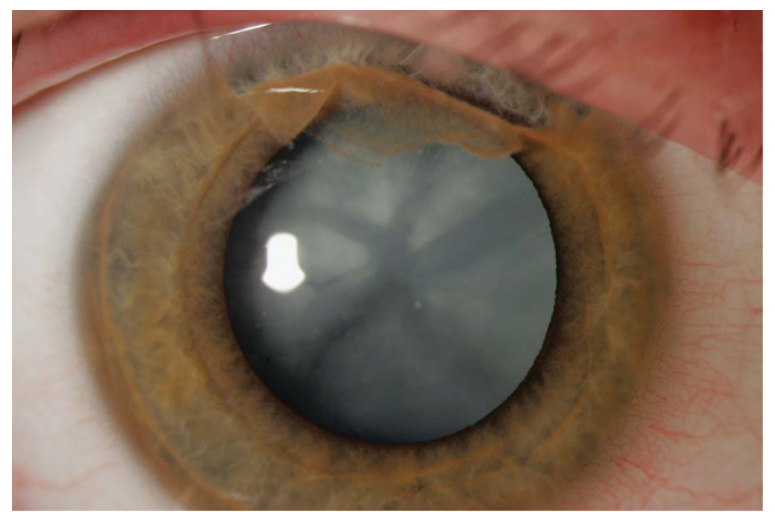

Figure 3 Iris changes secondary to trauma and dense cataract related to AD.

were no clinical signs of congenital anomaly or other iris related clinical disorders.

\section{Case 3}

A thirty-one-year-old Caucasian male presented with bilateral blurring of vision. There was a history of noninfectious, non-central, generalised lymphadenopathy secondary to severe AD. He had cataract surgery two years ago and five months ago in his right and left eye respectively at during presentation. Clinical examination revealed bilateral macula off $R D$ with a vision of 6/36 in the right eye and $\mathrm{CF}$ in the left eye. The right eye had two anterior irregular breaks close to the ora serrata with an infero-temporal RD involving the fovea. The left eye had three anterior horse shoe tears at the ora serrata with a bullous supero-temporal RD involving the fovea.

Bilateral PVR was noted during presentation and it was more extensive in his right eye. Bilateral simultaneous RD repair was carried out by PPV, cryopexy, and gas tamponade. Subsequently he developed recurrent $\mathrm{RD}$ twice in both eyes and had repair. RD in the right eye was related to PVR and new anterior breaks in the left eye.

Right eye needed removal of PVR membranes and silicone oil tamponade. Zonular abnormality was noticed intra-operatively the right eye. Management of this patient was further complicated by steroid response and glaucoma in the right eye. Visual acuity remained 6/36 in his right eye and $6 / 12$ in the left eye after recurrent RD repair surgery. Self inflicted trauma to eyes due to itching was described by this patient.

\section{Case 4}

Thirty-eight-year-old lady with very symptomatic AD, presented with a chronic inferior RD with two anterior breaks inferiorly in her left eye. She has bilateral cataract surgery at eighteen years of age. PPV/cryopexy and gas tamponade was performed to repair the RD. Corneal scarring in the left eye secondary to microbial keratitis made surgery more challenging due to the slight haze in the view of the surgical field. She developed recurrent RD needing repair with silicone oil. The retina detached under silicone oil secondary to PVR, requiring further surgery with removal of PVR membrane, retinectomy and re-insertion of silicone oil. Best corrected visual acuity with silicone oil in situ was 6/36 post surgery in the left eye. This patient also has bilateral subluxed IOL but not symptomatic. She is also a steroid responder.

\section{Case 5}

Forty four year old lady with severe $\mathrm{AD}$, was referred for management of dislocated IOL in the right eye, which is her only eye. She had bilateral cataract surgery two decades ago. Her left eye had a complex clinical course following cataract surgery for many years, which is discussed as below. She had low grade endophthalmitis post cataract surgery in the left eye. Clinical features were highly suggestive of Propionobacterium acnes related chronic endophthalmitis, although vitreous sampling was negative for organisms. She developed retinal detachment five months after cataract surgery and underwent combined PPV and scleral buckle procedure. Removal of the bag IOL complex was also performed during the retinal detachment repair, in view of the ongoing chronic low grade inflammation. A secondary scleral fixated IOL was placed, which dislocated subsequently. Further surgery was performed to place an anterior chamber IOL. Transient cystoid macular odema, steroid response, infection, and erosion of the buckle were added challenges in the management of the left eye. The patient was very symptomatic in her right eye, due to the dislocated lens implant and needed surgery. She had PPV and an iris sutured IOL in her right eye. With an episode of worsening of her $\mathrm{AD}$, she developed dislocation of the IOL into the vitreous cavity. Her management were complicated further by herpetic keratitis, glaucoma, and cystoid macular odema. She developed corneal decompensation and scarring. Combined PPV, removal of the dislocated implant and penetrating keratoplasty was done. She has a best corrected visual acuity of $6 / 18$ with ongoing visual rehabilitation in her right eye. In the mean time her vision in the left eye, has improved to 6/7.5 without any intervention for unknown reasons. Flare up of $\mathrm{AD}$ coincided with the dislocation of the IOL due to increased eye rubbing in this patient.

\section{Case 6}

A forty-nine-year-old gentleman presented with a retinal tear in his right eye, which was treated by laser 
retinopexy. Oral Cyclosporine was used for his AD and he also had asthma. Few months later, he had an episode of anterior uveitis with cystoid macular odema which resolved with topical steroids. During follow-up he had an epiretinal memebrane in his right eye. He had bilateral cataract surgery at the age of 30 .

Interestingly, the patient reported having abnormal shaped pupil in his left eye due to dislocation of the IOL with vitreous in the anterior chamber. He developed a macula off, temporal retinal detachment in his right eye due to a GRT, extending 180 degrees.

PPV, cryopexy, and gas tamponade was performed and vision recovered to $6 / 12$. The left eye needed PPV, removal of the lens bag complex and repositioning of the IOL by suturing it to the iris. Visual acuity improved to $6 / 6$ in his left eye. Three years later he developed dislocation of the IOL in his right eye and is awaiting surgical intervention for it. This patient has very severe symptoms due to $\mathrm{AD}$.

\section{Discussion}

$\mathrm{AD}$ is known to be associated with lid related problems, keratoconjuctivitis, keratoconus, and cataracts. ${ }^{4}$

Keratoconus is also associated with AD possibly due to eye rubbing. ${ }^{5} \mathrm{RD}$ secondary to $\mathrm{AD}$ was first reported in 1937. ${ }^{1}$ Several studies from Japan has described an association between AD and retinal detachment. ${ }^{6-8}$

In our observational case series of six patients with AD, eight out of twelve eyes had RD. This is the largest case series in Caucasian population in published literature in recent years although we included one Oriental patient. Various theories were postulated as a cause of RD related to AD. Balyeat suggested odema of the retina as a potential cause. ${ }^{1}$ Diseased vitreous was proposed as a cause for the retinal breaks by others. ${ }^{9}$ Oka et al compared the characteristics of RD secondary to trauma and AD and have concluded that ocular trauma secondary to eye rubbing can be a cause of RD in atopic patients. ${ }^{7}$ All our patients had a history of eye rubbing or tapping due to symptoms of itch. We support the theory of self inflicted trauma by eye rubbing and tapping as a potential cause for RD in this subgroup. The second patient in our series had iris changes, most likely due to self inflicted trauma by eye rubbing which can cause a dilemma in diagnosis due to the rarity of presentation.

Very often diagnosis of RD might not be easy. Retinal examination can be difficult due to corneal problems, thickened eyelids making indentation tough and also cataract may obscure the retinal details. There are two reported cases, where $\mathrm{RD}$ in $\mathrm{AD}$ can masquerade as panuveitis with a rapidly progressive cataract. ${ }^{10}$ In one study, authors have demonstrated that retinal breaks are more common in the vitreous base border in the upper temporal quadrant in $\mathrm{RD}$ associated with $\mathrm{AD} .^{7}$ In a multicentre study, the initial reattachment rate was $75.3 \%$ and the final reattachment rate was $92.6 \%$ in RD repair in this condition. ${ }^{8}$ Surgical reattachment in this type of RD is lesser than a simple rhegmatogenous RD due to presence of PVR at the time of presentation.

In our series, two patients had GRT and others had very anterior breaks close to the ora serrata (Table 1). Two patients with GRT had the most severe AD in our series. None of them had retinal dialysis. Six patients had temporal $\mathrm{RD}$, one had an inferior detachment and the nature of the break was not known in one patient. Retinal breaks were identified correctly in three eyes before surgery; breaks in the other four eyes were identified intra-operatively by indentation due to the anterior nature of the breaks. PVR was found at time of presentation in five eyes, making surgical management difficult. Epiretinal memebrane developed following RD repair surgery in one patient. Recurrent RD occurred in three eyes in this series and all had PVR during presentation. Two of these three needed silicone oil tamponade. Cataracts co-exist with this condition in young patients and appropriate management is essential. All patients in our series needed cataract surgery between the age of 18 and 31. All our patients had axial lengths within normal limits. Keratoconus was ruled out in two patients with astigmatism by corneal mapping. Astigmatism was minimal negating the need for a toric IOL or surgical correction during cataract surgery for those patients. A multifocal IOL was used in case two as the patient was young, had never worn glasses and at presentation had no history or concerns relating to retinal detachment. The IOL has been effective giving the patient both distance and reading vision and has not posed a problem with subsequent surgery. In this unique group of patients with high risk of subsequent RD and IOL subluxation, multifocal IOLs are probably best avoided. Before detachment repair surgery, the intra-ocular pressure was low in all eyes due to the retinal detachment and none of them had inflammation in the anterior chamber.

Schwartz-Matsuo syndrome was not encountered in our case series. Bilateral RD was present in two patients at initial presentation and one needed simultaneous RD repair surgery. Another patient needed bilateral simultaneous surgery with RD repair in one eye and cataract surgery in the second eye. A detailed discussion regarding the risks and benefits with the patient is very important before embarking on bilateral simultaneous surgery. Three of our patients were steroid responders and two developed glaucoma over a period of few years. There is no known association between steroid response glaucoma and AD in the published English literature.

Late dislocation of the IOL is seen in six eyes in our series and this is most likely due to constant trauma by 
Table 1 Characteristics of retinal break, RD, subluxation of IOL and history of eye rubbing

\begin{tabular}{|c|c|c|c|c|c|}
\hline Eye rubbing & Cataract surgery & Location of $R D$ & Nature/location of break & PVR at initial presentataion & Subluxation of IOL \\
\hline Y & $\mathrm{N}$ & Temporal & Ora serrata & Y & $\mathrm{N} / \mathrm{A}$ \\
\hline Y & $\mathrm{Y}$ & Temporal & Ora serrata & $\mathrm{Y}$ & $\mathrm{N}$ \\
\hline Y & $\mathrm{Y}$ & Superotemporal & GRT & $\mathrm{N}$ & $\mathrm{N}$ \\
\hline Y & $\mathrm{Y}$ & Inferotemporal & Ora serrata/irregular & Y & $\mathrm{N}$ (zonule weakness) \\
\hline Y & $\mathrm{Y}$ & Superotemporal & Ora serrata/HST & Y & $\mathrm{N}$ \\
\hline Y & $\mathrm{Y}$ & Inferior & Ora serrata & $\mathrm{Y}$ & Y \\
\hline $\mathrm{Y}$ & $\mathrm{Y}$ & Not known & Not known & $\mathrm{N}$ & $\mathrm{Y}$ \\
\hline $\mathrm{Y}$ & $\mathrm{Y}$ & Temporal & GRT & $\mathrm{N}$ & $\mathrm{Y}$ \\
\hline
\end{tabular}

Abreviations: HST, Horse shoe tear; N, No; Y, Yes.

rubbing the eyes. There is only one case report about IOL dislocation in $\mathrm{AD}$ in the published literature. ${ }^{11}$ This study is the first in literature to report six eyes with similar condition. In case three of our series, abnormality in zonules were noticed during PPV, although no clinical subluxation of IOL was seen.

We reported our case series in ascending order of age in order to reveal the extent and progressive nature of the ocular problems with increasing age. These patients need cataract surgery earlier in life and dislocation of the IOL occurs at later age. RD may occur at any time. The incidence of post cataract surgery RD in atopic cataracts may be reduced by IOL implantation in the bag with no intra operative posterior capsule rupture. ${ }^{12}$ Decrease in $\mathrm{RD}$ related to $\mathrm{AD}$ in the last decade has been reported and this is thought to be due to better control of dermatitis. ${ }^{13}$ $\mathrm{RD}$ associated with $\mathrm{AD}$ is not widely reported in Caucasians although the prevalence of AD is not low in Caucasians. Mutation of the gene encoding for the protein Filaggrin is known to be a predisposing factor for developing AD. ${ }^{14}$ A difference in the spectrum of mutations between European and Asian population has been reported. ${ }^{14}$ Genetic variations might be a reason for difference in the RD rate associated with AD and severity in different population.

In conclusion, this study illustrates that self inflicted trauma by eye rubbing is most likely a factor in the causation of retinal detachment and subluxation of IOL in AD. It is the repetitive nature of the action or behaviour over a long period of time that gives rise to cumulative damage or trauma resulting in these observed changes. Temporal retina is involved more commonly in this type of RD. GRT might be associated with very severe AD. Majority of the breaks were located anteriorly, close to the ora serrata. PVR is often present during initial presentation. Co-existing cataract, corneal pathology, steroid response, and cystoid macular odema can make the management more complex. Late recurrent dislocation of IOL in AD has been identified by this study. More awareness and close working relationship is needed among ophthalmologists and dermatologists regarding this rare association between $\mathrm{AD}, \mathrm{RD}$ and late dislocation of IOL.

\section{Summary}

What was known before

- $\mathrm{RD}$ is associated with $\mathrm{AD}$.

- Eye rubbing is most likely a factor in the causation of $\mathrm{RD}$ in $\mathrm{AD}$.

What this study adds

- PVR is often present in RD associated with AD.

- Late dislocation of IOL may occur in AD.

- $\mathrm{RD}$ and late dislocation of IOL in AD could be related to eye rubbing.

\section{Conflict of interest}

The authors declare no conflict of interest.

\section{Acknowledgements}

We would like to thank Ann Ramsey our ophthalmic photographer and medical illustrations department at our institution for providing the relevant clinical pictures.

\section{References}

1 Balyeat RM. Complete retinal detachment (both eyes): with special reference to allergy as a possible primary etiologic factor. Am J Ophthalmol 1937; 20: 580-582.

2 Ingram RM. Retinal detachment associated with atopic dermatitis and cataract. Br J Ophthalmol 1965; 49: 96-97.

3 Simpson CR, Newton H, Hippisley-Cox J, Sheikh A. Trends in the epidemiology and prescribing of medication for eczema in England. J R Soc Med 2009; 102(3): 108-117.

4 Eiseman AS. The ocular manifestations of atopic dermatitis and rosacea. Curr Allergy Asthma Rep 2006; 6(4): 292-298.

5 Bawazeer AM, Hodge WG, Lorimer B. Atopy and keratoconus: a multivariate analysis. Br J Ophthalmol 2000; 84(8): 834-836.

6 Takahashi M, Suzuma K, Inaba I, Ogura Y, Yoneda K, Okamoto $\mathrm{H}$. Retinal detachment associated with atopic dermatitis. Br J Ophthalmol 1996; 80(1): 54-57. 
7 Oka C, Ideta $\mathrm{H}$, Nagasaki $\mathrm{H}$, Watanabe K, Kouichi Shinagawa I. Retinal detachment with atopic dermatitis similar to traumatic retinal detachment. Ophthalmology 1994; 101: 1050-1054.

8 Hida T, Tano Y, Okinami S, Ogino N, Inoue M. Multicenter retrospective study of retinal detachment associated with atopic dermatitis. Jpn J Ophthalmol 2000; 44(4): 407-418.

9 Coles RS, Laval J. Retinal detachments occurring in cataract associated with neurodermatitis. Arch Ophthalmol 1952; 48: 30-39.

10 Lim WK, Chee SP. Retinal detachment in atopic dermatitis can masquerade as acute panuveitis with rapidly progressive cataract. Retina 2004; 24(6): 953-956.
11 Yamazaki S, Nakamura K, Kurosaka D. Intraocular lens subluxation in a patient with facial atopic dermatitis. J Cataract Refract Surg 2001; 27(2): 337-338.

12 Inoue M, Shinoda K, Ishida S, Uchida A, Kurosaka D, Katsura H, Tsubota K. Intraocular lens implantation after atopic cataract surgery decreases incidence of postoperative retinal detachment. Ophthalmology 2005; 112(10): 1719-1724.

13 Sasoh M, Mizutani H, Matsubara H, Furuta M, Matsui Y, Yamanaka K, Kondo M. Incidence of retinal detachment associated with atopic dermatitis in Japan: review of cases from 1992 to 2011. Clin Ophthalmol 2015; 9: 1129-1134.

14 Osawa R, Akiyama M, Shimizu H. Filaggrin gene defects and the risk of developing allergic disorders. Allergol Int 2011; 60(1): 1-9. 